\title{
Ueber Resorption durch die Lungen
}

\author{
On Resorption in the Lungs
}

Autor

Institut

\section{R. Kropp}

Deutsches Tuberkulose-Archiv

\section{Bibliografie}

DOI http://dx.doi.org/

10.1055/s-0030-1256085

Online-Publikation: 12.1.2011

Pneumologie 2011; 65:

602-606 @ Georg Thieme

Verlag KG Stuttgart · New York ISSN 0934-8387

\section{Korrespondenzadresse}

Dr. med. Robert Kropp

Liegnitzer Straße 5

36100 Petersberg

dr.robert.kropp@gmx.de

\section{Prolog \\ $\nabla$}

Im Folgenden wird den geehrten Lesern des Historischen Kaleidoskops der Text einer Dissertation aus dem Jahre 1886 zur Lektüre angeboten: „Ueber Resorption durch die Lungen“. Die Dissertations-eigentümlichen Passagen (Lebenslauf, Danksagungen etc.) wurden entfernt, ebenso elf der ursprünglich zwanzig Versuchsbeschreibungen, um den Text lesbarer und möglichst kurzweilig zu halten. Alle Experimente mit intratrachealer Applikation der Chemikalien wurden jedoch belassen.

Thema und Inhalt der Arbeit sind recht witzig, die Sprache ist zeitgebunden. Und so mag dieser Text das Wissen des Lesers vermehren, und das mit einigem Vergnügen.

Dr. Robert Kropp
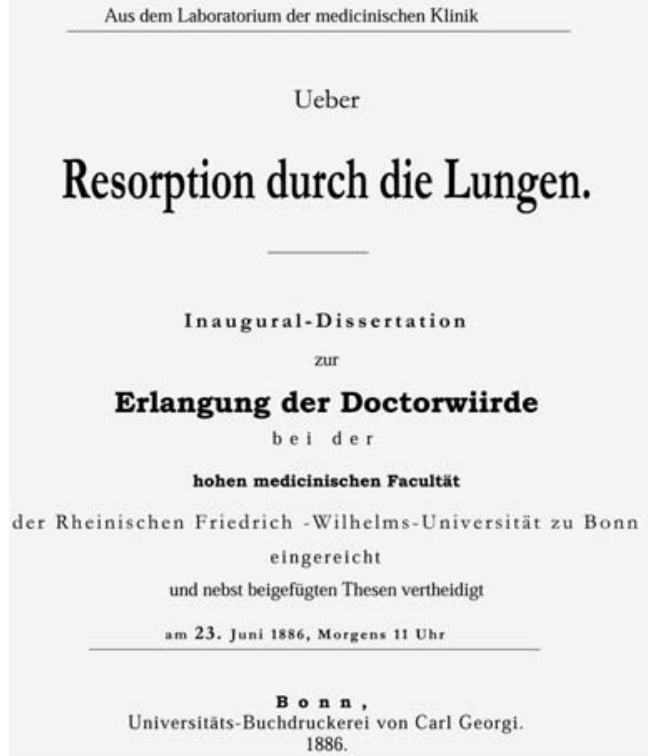

Wenn man bei dem heutigen Standpunkte der medizinischen Wissenschaften annimmt, dass eine grosse Anzahl von Krankheiten durch Infection von aussen her in den Körper eingeschleppt wird, so scheint es nicht unberechtigt, die Resorptionsgeschwindigkeit von seiten der Lungen etwas näher in den Kreis der Betrachtung zu ziehen, zumal die Lungen als eines der Hauptorgane des Organismus für die Aufnahme infectiöser Stoffe anzusehen sind.

Es liegt nicht in dem Rahmen dieser Arbeit, auf die Controversen einzugehen, welche lange Zeit darüber bestanden, auf welchem Wege überhaupt in die Lungen eindringende Stoffe ihren Uebertritt in den Saftstrom und also in den Organismus bewerkstelligten, ebensowenig zu entscheiden, wie weit den Lymphgefässen die Hauptbedeutung für diesen Vorgang zukomme, oder den Blutgefässen allein, oder endlich beiden Gefässsystemen gemeinschaftlich. Vielmehr bezweckt die experimentelle Studie darzuthun, wie ungemein rasch von seiten der Lunge die verschiedensten Stoffe resorbirt werden.

Die Resorption von Stoffen ist im Allgemeinen abhängig von verschiedenen Momenten; unter denselben beansprucht eine einschneidende Bedeutung der jeweilige Aggregatzustand des aufzunehmenden Körpers. In der That lassen sich hier ganz bestimmte Differenzen erkennen, so dass auch hier die Eintheilung der resorptionsfähigen Stoffe nach ihrer physikalischen Eigenthümlichkeit in feste, flüssige und gasförmige Körper zu Recht bestehen muss. Dieser Disposition sind auch die Arbeiten gefolgt, welche über die Resorptionsverhältnisse von seiten der Lungen Aufschluss geben sollten. Eine grosse Anzahl von Arbeiten nämlich liegen vor, welche sich mit den Resorptionsverhältnissen fester und gasförmiger Körper durch die Lungen beschäftigen. Die darauf 
bezügliche Literatur ist in erschöpfender Weise gesammelt in der neuesten Arbeit von Arnold $^{1}$ ); als Anhang dieses beachtenswerten Werkes finden wir nicht weniger wie 140 Arbeiten angeführt, die sich mehr oder weniger mit Inhalation fester Bestandtheile, speciell mit Staubinhalation und Staubmetastase befassen, 264 Nummern im Garnen, die über die Resorption fester oder gasförmiger Körper durch die Lungen handeln.

Auffallend ärmlich muss es im Gegensatz zu dieser reichen Litteraturblüthe erscheinen, wenn nur sehr wenige Arbeiten vorliegen, welche die Resorptionsverhältnisse flüssiger Körper durch die Lungen in Betracht ziehen.

Soweit Quellen über diesen Gegenstand von uns durchforscht werden konnten, handelt es sich wesentlich nur um zwei Arbeiten, auf welche wir aus dem Grunde näher eingehen, weil es in unserer Intention liegt, Versuche mitzutheilen, welche zu diesen Versuchen in engster Verwandtschaft stehen. J. Wasbutzky²) war der erste, der hierüber 1879 Untersuchungen veröffentlichte. Das Wesentlichste seiner Ergebnisse führen wir in Folgendem an.

W. verfolgt in seiner Arbeit zwei Zwecke. Erstens sucht er den Beweis zu bringen, dass die Resorption in den Lungen früher von den Lymphbahnen als von den Blutgefässen aus geschähe. Zu diesem Zwecke spritzte er Kaninchen gewisse Lösungen vermittelst einer Pravazschen Spritze in die blossgelegte Trachea; er prüfte dann gleichzeitig den durch einen Katheter entleerten Harn und aus der Carotis externa entnommes Blut auf die Anwesenheit des injicirten Stoffes. Im Verlauf seiner Experimente will er den Nachweis für seine oben aufgestellte Behauptung dadurch führen, dass er das Vorhandensein des eingespritzten Stoffes im Harn früh er als im Blut zu konstatiren glaubt. Wir lassen dahingestellt, wie weit man aus solchem Verfahren bindende Schlüsse ziehen darf, wir müssen aber darauf aufmerksam machen, dass nicht alle Fehlerquellen beseitigt sind, aus denen unwillkürlich unrichtige Schlussfolgerungen hervorgehen müssen. Damit der Leser sich hinreichend orientiren kann, führen wir ein Beispiel an:

Nach der Aufzählung und Beschreibung seiner Versuche mit Atropin schliesst W. ohne Weiteres, letzteres sei bereits in den Harn übergegangen zu einer Zeit, als im Blut nicht eine Spur Atropin zu finden gewesen wäre. Sehen wir uns die Versuche im Detail an, so finden wir, dass unter den 13 mit Atropin angestellten Thierversuchen 7 Experimente vorliegen, in denen W. weder im Harn noch im Blut Atropin nachweisen konnte; in 2 Fällen wies er gleichzeitig im Blut und Harn Atropin nach, in einem Falle nur im Harn, in einem andern nur im Blut, in 2 Fällen untersuchte er das Blut überhaupt nicht. Warum nun aus solchem Ergebniss W. die These aufstellen zu dürfen glaubt, dass seine oben citirte Ansicht hierdurch bewiesen sei, ist uns nicht recht ersichtlich, es lässt sich vielmehr nach unserer Ansicht aus diesem so verschiedenartigen Resultate kein bestimmtes Gesetz ableiten, am wenigsten aber das, was W. glaubt.

Der zweite Hauptzweck der Arbeit Wasbutzky's war der, den Termin zu bestimmen, bis zu welchem ein Teil der eingeführten Stoffe unzweifelhaft in die Blutmasse übergegangen sei; für die einen Substanzen genügt der Nachweis ins Harn und im Blute, für die anderen wird die eingetretene Resorption durch die auftretenden Vergiftungserscheinungen nachgewiesen. Der Autor geht nicht darauf ein, absolute Bestimmungen über den zeitlichen Verlauf der Resorptionsgeschwindigkeit zu machen, sondern er beschränkt sich mehr auf eine comparative Bestimmung derselben. Aber auch hier müssen wir auf einige Fehler hinwei-

\footnotetext{
${ }^{1}$ Arnold: Untersuchungen über Staubinhalation und Staubmetatase 1885.

2 J. Wasbutzky: Ueber Resorption durch Lungen. Inaug.-Dissert. Königsberg 1879 .
}

sen, die der Arbeit anhaften, und die gerade die Berechtigung gaben, einen Theil der Experimente Wasbutzky's zu wiederholen und zu controlliren.

Zunächst gebrauchte W. kurze Zeit nacheinander dieselben Versuchsthiere zu verschiedenen Experimenten, sodass er selbst z. B. nach Einspritzung von Chloralhydrat und Eisenchlorid in die unangenehme Lage kam, nicht beweisen zu können, ob die Folgezustände von der Injection des einen oder anderen Stoffes herrührten. Sodann scheint es uns ungenau zu sein, dass bei verschiedenen Versuchen in gewissen Zeitabschnitten Injectionen verschiedene Male nacheinander wiederholt werden, ohne die Zeit, welche zwischen den einzelnen Injectionen liegt, anzugeben, sodass eigentlich von einer, wenn auch nur ungefähren Bestimmung der Zeit, in welcher die Resorption sich vollzog, gar keine Rede sein konnte.

Als Resumé der übrigens sehr fleissigen Arbeit, die nicht weniger als 70 Versuche aufweist, wird mit Recht constatirt, dass die Resorption von seiten der Lungen eine ungeheuer rasche ist, rascher als die vom Magen aus, mindestens ebenso rasch, wenn nicht noch rascher sich vollzieht als die vom Unterhautszellgewebe aus.

Der Vollständigkeit halber fügen wir dann an, dass lange vor W. gelegentlich darauf hingewiesen war, mit welcher Schnelligkeit die Lungen flüssige Massen resorbirten, nämlich von einem französischen Autor Dr. Auphan ${ }^{3}$, der diese Beobachtung bei der Untersuchung der Wirkungen des Mineralwassers des Bades Euzet machte.

Wenden wir uns jetzt zu der andern Arbeit, welche von Peiper ${ }^{4}$ ) 1884 veröffentlicht, in erster Linie auf den Versuchen W. fusst.

Peiper wiederholte zunächst einige Versuche W.'s, besonders solche, bei denen W. zu keinem eigentlichen Resultate gelangt war, z.B. Injectionen mit HaemoglobinLösung, bei denen es W. nicht gelungen war, den Blutfarbstoff im Harn nachzuweisen.

P. bemüht sich dann weiter, abgesehen von einigen kleinen Versuchen unter normalen Verhältnissen, die Resorptionsfähigkeit der Lungen auch unter pathologischen Verhältnissen einer näheren Untersuchung zu unterziehen. „Einerseits hoffte er, durch die Ausschaltung bestimmter Nerven den Einfluss derselben auf die Resorptionsvorgänge kennen zu lernen, andererseits bestand die Möglichkeit, dass die in gewisse pathologische Verhältnisse gebrachten Lungen ein abweichendes Verhalten von der bisher beobachteten Resorptionsthätigkeit zeigen würden.“

Er durchschnitt deshalb die einzelnen Nerven nach der Reihe, die auf die Resorptionsthätigkeit der Lungen einen etwaigen Einfluss auszuüben im Stande gewesen wären, den vagus, sympathicus und phrenicus, und spritzte den Thieren Lösungen von der Trachea aus in die Lungen. In keinem Falle jedoch gelang es ihm, von den normalen Verhältnissen abweichende Resultate zu erzielen, und in keinem Falle konnte ein Einfluss der durchschnittenen Nervenbahnen auf die Resorptionsvorgänge nachgewiesen werden. Ebenso blieben die Resorptionsverhältnisse der Lungen ganz dieselben, wenn er bei fiebernden oder in asphyktischen Zustand versetzten Thieren die Injection von der Luftröhre aus machte. Es liess sich kein Unterschied von den normalen Verhältnissen ausfindig machen. Die Thatsache, dass die Wirkung in die Lungen injicirter Gifte bei Thieren, denen er vorher eine pneumonische Infiltration durch doppelseitige Vagusdurelischnei dung erregt hatte, eine raschere sei, glaubt P. nur dem heruntergekommenen Zustand der Thiere zuschreiben und keineswegs

\footnotetext{
${ }^{3}$ Gazette Médicale de Paris. 1861. S. 697.

${ }^{4}$ E. Peiper: Ueber Resorption durch die Lungen. Zeitschr. f. Kl. Med. 1884. S. 293.
} 
als die Aeusserung einer beschleunigten Resorption auflassen zu müssen. Es zeigt hierdurch P., dass in seinen pathologischen Fällen ein anderes Verhältniss der Resorptionsvorgänge wie bei normalen Lungen nicht existirt.

Die von uns selbst angestellten Experimente, welche in dem Laboratorium der medizinischen Klinik ausgeführt wurden, beschäftigen sich hauptsächlich damit, zu eruiren: „In welcher Zeit erfolgt der Uebertritt resorptionsfähiger, flüssiger Stoffe durch die Lungen in den Organismus?"

Als Versuchsthiere benutzten wir für unsere Untersuchungen Kaninchen. Den Thieren, welche aufgeschnallt werden, wird die Trachea freigelegt, und in derselben der Länge nach ein etwa $1 \mathrm{~cm}$ langer Einschnitt gemacht. (W. injicirte nur vermittelst einer Pravaz'schen Spritze, P. bediente sieh beider Methoden.) Sodann wird eine Spritze mit langem Ansatzrohr durch die Oeffnung in die Trachea eingeführt, und die betreffende Flüssigkeit direct in die Lungen eingespritzt. Die Lösungen waren jedesmal bis auf normale Körpertemperatur erwärmt, da sie dann besser von den Thieren vertragen wurden, als kalte Flüssigkeiten, die sehr oft einen heftigen Hustenreiz erregten. Vor dem Eingiessen der zu prüfenden Substanz durch die geöffnete Luftröhre wird ein elastischer Katheter in die Blase des Versuchsthieres eingeführt (wir benutzten meistens Männchen, die uns zu diesem Zwecke geeigneter schienen), um den abfliessenden Harn sofort auffangen und zeitlich abgrenzen zu können. Die Uretheren zu diesen Versuchen benutzen zu können, geht nicht an, wie dies Wasutzky schon gefunden hat, und wie wir es bestätigen können. Peiper hatte bereits darauf aufmerksam gemacht, dass die Resorption schneller vor sich geht, wenn das Thier während des Versuches aufgerichtet wird, als wenn es horizontal gelagert ist. Er sucht den Grund hierzu darin, dass sich bei der senkrechten Stellung des Thieres die Flüssigkeit rascher bis in die kleinsten Bronchien verbreiten könnte, und so eine grössere Resorptionsfläche vorhanden sei. Unsere Experimente bestätigen seine Ansicht in dieser Richtung vollkommen. Wahrscheinlich spielt bei der aufrechten Stellung des Thieres die Schwerkraft der injicirten Flüssigkeit selbst eine Rolle.

In demselben Augenblicke, in welchem dem Thiere die Injection in die Trachea von uns gemacht wird, lassen wir den Harn durch den Katheter abfliessen; der Harn träufelt meistens tropfenweise langsam ab, sodass der Eintritt der Reaction auf die injicirte Substanz fast auf die Sekunde bestimmt werden kann. Selbstverständlich sind die so gefundenen Zeitwerte, welche die Reaction im Harn ergiebt, grösser als die Resorptionsgeschwindigkeit in den Lungen; die Resorption ist offenbar noch viel schneller als die gefundenen Zahlen angeben, weil zunächst der injicirte Stoff einen Teil des Kreislaufs mitmacht, um erst in die Nieren gelangen zu können, und der Weg, den der Harn braucht, um von den Nieren durch die Uretheren in die Harnblase zu gelangen, doch immerhin eine gewisse Zeit in Anspruch nimmt. Rechnen wir nun die Zeit, welche der Harn braucht, um in die Blase zu kommen, von unseren Zahlen ab, so werden unsere Experimente Resultate ergeben, dahinzielend, dass von Seiten der Lunge die von uns geprüften Stoffe ungemein rasch aufgesaugt worden sind. Gleichzeitig verbinden wir mit dieser Untersuchungsweise vergleichende Bestimmungen, wie rasch die Resorption im Magen und vom Unterhautszellgewebe aus sich im Verhältniss zu der durch die Lungen erfolgten bewerkstelligt. Wir lassen jetzt die Versuche selbst folgen.

\section{Versuch 1. \\ $\nabla$}

\section{Morgens 11 Uhr.}

Einem mittelgrossen Kaninchen werden vermittelst einer Spritze $5 \mathrm{cbcm}$ einer $5 \%$ igen Natrium-salicylicumlösung durch die in der Trachea gemachten Oeffnung in die Lungen eingegossen. Als Reagens für den Nachweis der Salicylsäure im Harn dient Liquor ferri sesquichlorati. 54 Sekunden nach der Injection des Natrium salicylicum in die Lungen zeigt der abfliessende Harn die erste Reaction auf Salicylsäure und zwar ziemlich stark, indem eine deutliche blaue Färbung beim Niederträufeln des Harns auf die Liquorferrilösung hervorgerufen wird. Die Intensität der Reaction nimmt zu, bis sie nach 27 Minuten denselben Stärkegrad erreicht, als die durch Verbindung der reinen 5\%igen Natrium-salicylicum- und Liquor-ferrilösung entstandene Reaction aufweist. Die Stärke der Reaction lässt 31 Minuten nach der Injection nach. Das Versuchsthier bleibt bei dem Versuche völlig ruhig und zeigt keine dyspnoischen Erscheinungen. Am folgenden Morgen (9 Uhr) ist eine, wenn auch schwache Reaction noch vorhanden. Um 12 Uhr ist der Nachweis einer Reaction nicht mehr zu erbringen.

$$
[\ldots]
$$

\section{Versuch 4. \\ $\nabla$}

Morgens 11 Uhr.

Einem grossen Kaninchen wird eine Pravaz'sche Spritze einer 25\%igen Natrium-salicylicumlösung subcutan eingespritzt, sodass das Thier genau dieselbe Dosis Natrium salicylicum erhielt, wie in den obigen Versuchen. Erst 8 Minuten nach der Injection zeigt sich im Harn nach Zusatz derselben Liquor-ferrilösung eine schwache Reaction, die nur sehr langsam an Stärke zunimmt. Am folgenden Morgen war eine Reaction nicht mehr bemerkbar.

Versuche über die zeitliche Ausscheidung der Salicylsäure oder des Natrium salicylicum nach Einverleibung per os in den Körper anzustellen, scheint uns müssig zu sein, da eine Menge von Versuchen betreffs dieses Gegenstandes vorliegen. Nach Fleischer ${ }^{5}$ soll beim Menschen erst nach 1 bis $1 \frac{1}{2}$ Stunden bei einer Dosis von 5,0 gr die Ausscheidung durch den Harn vor sich gehen. Diese Zeit möchte doch etwas zu lang angenommen sein; wir machten an uns selbst einen derartigen Versuch, indem Morgens $1 \mathrm{gr}$ Natrium salicylicum in den nüchternen Magen gebracht wurde. Jedoch liess sich bereits 25 Minuten später Salicylsäure im Harn nachweisen. Nach Feser ${ }^{6}$ sollen Pflanzenfresser die per os aufgenommene Salicylsäure rascher ausscheiden wie Fleischfresser. Jedenfalls ist die Differenz bei beiden nicht so gross, dass wir Zahlen über die vom Magen aus stattgefundene Resorptionsgcscbwindigkeit vorfinden, wie sie uns bei der Resorption von seiten der Lungen aus entgegentreten.

\section{Versuch 5.}

$\nabla$

Es werden einem grossen Kaninchen $5 \mathrm{cbm}$ einer $2 \%$ igen Chininum-muriaticumlösung in die Lungen von der Trachea aus eingebracht. Als Reagens zum Nachweis des Chinins im Harn bedienen wir uns der Phosphormolybdänsäure.

\footnotetext{
${ }^{5}$ Nothnagel-Rossbach: Lehrbuch der Arzneimittellehre S. 446.

${ }^{6}$ Nothnagel-Rossbach: Lehrbuch der Arzneimittellehre S. 446.
} 
Die Cautelen, welche bei der Anwendung dieser Säure als Reagens auf Chinin im Harn betrachtet werden müssen, haben wir nicht aus dem Auge gelassen. Zunächst kommt die Fällung von Kreatin, Kreatinin, Xanthin, Guanin und Sarkin durch die oben erwähnte Säure nicht in Betracht, da wir die Experimente nur mit Pflanzenfressern vornahmen. Coffein kommt selbstverständlich ebenfalls nicht in Betracht. Kalisalze, nicht aber Natron und Lithionsalze, sollen nach Debray ${ }^{7}$ in Verdlinnung von (K0):500 durch die Phosphormolybdänsäure gefällt werden. Kerner ${ }^{8}$ hat jedoch in den im Harn durch dieses Reagens bewirkten Niederschlägen niemals Alkalimetalle aufgefunden. Harnstoff, Harnsäure, Hippursäure und Sarkonin werden durch unser Reagenz nicht gefällt.

Es könnte also nur noch die Fällung von Eiweiss in Frage kommen. Wir haben uns jedoch selbst vor dem Versuch vergewissert, dass der Harn vollständig eiweissfrei sei, und zwar durch mehrere Proben.

Eine Minute 5 Sekunden nach der Injection der Chininlösung zeigt sich bei Zusatz des Reagens im Harn ein schwacher Niederschlag, der beim Stehen deutlicher wird. Nach 21/2 Minuten ist die Reaction sehr deutlich. Nach 5 Minuten bildet sich sofort nach Zusatz des Reagens im Harn ein dickflockiger, grünlicher Niederschlag. Das Thier stirbt 10 Minuten nach der Injection des Chinins. Aus der Harnblase bei der Section genommener Harn ergiebt bei Zusatz von nur einem Tropfen der Säure einen ungemein starken Niederschlag.

$$
[\ldots]
$$

\section{Versuch 7.}

$\nabla$

Einem grossen Kaninchen werden von' der Trachea aus 5 cbm einer 5\%igen Chininum-morphum-boricumläsung in die Lungen eingebracht.

Da das Chin. amorph. bor. ${ }^{9}$ kein echtes Chininalkaloid ist, untersuchten wir vor dem Experiment die Reaction auf dasselbe vermittelst der Phosphormolybdänsäure, die uns bei dem Nachweis des Chininum muriaticum im Harn gute Dienste geleistet hat. Dieselbe ist nach unsern Versuchen nur in beschränkter Weise brauchbar, schon bei einer Lösung des Chin. amorph. bor. von $1: 10000$ Wasser ist keine Spur einer Reaction bei Zusatz von Phosphormolybdänsäure zu sehen. Erst nach mehrstündigem Stehen erscheint in dem Reagensgläschen, in dem wir die Lösung stehen liessen, ein weisslicher Niederschlag. Wir haben deshalb das Reagens zu Hülfe gezogen, das Schwengers bei seinen Versuchen im Binz'schen Laboratorium zum Nachweise des Chin. mur. angewandt hat: 8 Jod +4 Kal. jod. auf 160 Wasser, mit Zusatz eines Tropfens verdünnter Schwefelsäure. Das Resultat, das wir damit erzielt haben, ist ein sehr günstiges zu nennen, weil wir das Chin. amorph. bor. noch in einer verdünnten Lösung von 1:60000 vermittelst dieses Reagens nachweisen konnten.

Das Eingiessen der Lösung in die Lungen vollzieht sich glatt, so dass der gesammte Inhalt der Spritze in dieselben entleert wird. 21ํㄹ Minuten nach der Injection treten Krämpfe auf, es stellt sich Athemnot ein. Der Harn wird in Reagensgläsern aufgefangen. Das Thier entleert schon auf leisen Druck hin sehr flott Harn.

\footnotetext{
${ }^{7}$ Buna. mem. de la societe de Paris. 1866. S. 404.

${ }^{8}$ Kerner: Beiträge zur Kenntniss der Chininresorption. Pflüger's Archiv. 2. Jahrgang.

${ }^{9}$ Näheres über das Chinin. amorph. bor.: Finkler u. Prior: Mittheilungen über d. Chinin. am. b. Deutsche Med. Wochenschr. 10. Jahrg. Nr. 6. S. 81.
}

Der $1 / 2$ Minute nach der Injection aufgefangene Harn ergiebt keine Reaction, am Ende der ersten Minute ist eine schwache Reaction erkennbar, die nach 11/2 Minuten sehr stark wird. 7 Minuten nach der Injection stirbt das Thier. Die Section ergiebt nichts Besonderes. Der in der Harnblase bei der Section befindliche Harn zeigt ebenfalls einen starken Gehalt an Chin. amorph. bor.

$[\ldots]$

\section{Versuch 10. \\ $\nabla$}

Einem mittelgrossen Kaninchen werden $5 \mathrm{cbcm}$ einer 5\%igen Jodkaliumlösung von der Trachea aus in die Lungen injicirt. Zum Nachweis des Jods im Harn benutzen wir Stärkekleister und rauchende Salpetersäure.

Obwohl sonst der Schwefelkohlenstoff beim Nachweis des freien Jods bessere Dienste leistet, so eignet sich nach den Erfahrungen, die in hiesiger Klinik von Prior gemacht sind, und die in der neuesten Arbeit Sticker's ${ }^{10}$ ihre Bestätigung finden, für den Nachweis des Jods oben angegebene Reaction besser, zumal sie bei Verdünnungen von 1:360000 noch vollständig Jod nachzuweisen im Stande ist.

$$
[\ldots]
$$

\section{Versuch 13. \\ $\nabla$}

Einem mittelgrossen Kaninchen werden $5 \mathrm{cbm}$ einer $10 \%$ igen Ferrocyankaliumlösung von der Trachea aus in die Lungen injicirt. Zum Nachweis im Harn dient eine Liquor-ferri-sesquichloratilösung. Kurz vor der zweiten Minute nach der Injection war eine schwache Reaction im Harne sichtbar, die sich durch einen grünlich-bläulichen Schimmer erkennen lässt. Nach 21ํㄹ Minuten wird die Reaction sehr deutlich. Nach 20 Minuten entsteht nach Zusatz eines einzigen Tropfens des Reagens ein hellblauer Niederschlag im Harn, der nach 50 Minuten eine dunkelblaue Färbung annimmt. Die Intensität der Reaction lässt 60 Minuten nach der Injection wieder nach. Das Thier zeigt während des Versuches und auch nachher keine Zeichen von Dyspnoe oder Krampfanfällen.

Während wir in den bisher angeführten Versuchen den Nachweis für den Zeitpunkt der durch die Lungen stattgehabten Resorption durch Auftreten der injicirten Substanz in dem Harn zu constatiren gesucht haben, können wir bei den jetzt folgenden Versuchen die von Seiten der Lungen erfolgte Resorption durch die bei den verschiedenen 'Tieren auftretenden Vergiftungserscheinungen nachweisen. Wir werden sehen, dass die Resultate mit den oben gewonnenen fast ganz übereinstimmen.

\section{Versuch 14.}

Es werden einem Kaninchen (1990 gr schwer) 5cbcm einer 0,01\% igen Strychninurn-sulfuricumlösung von der Trachea aus direct in die Lungen eingespritzt, sodass das Thier im Ganzen 0,0005 cbcm Strychnin erhält. 65 Sekunden nach der Injection lösen Erschütterungen des Brettes, auf dem das Thier aufgeschnallt ist, heftige tetanische Krämpfe aus. 2 Minuten nach der

\footnotetext{
${ }^{10}$ Sticker: Untersuchungen über die Elimination des Jodes im Fieber. Berl. Klin. Wochensch. 1885. Nr. 35
} 
Injection entstehen starker Opistotonus und exquisite Krämpfe, die Athmung sistirt. 23/4 Minuten nach der Injection beginnt die Athmung wieder langsam, der Tetanus lässt nach und ist nach der 4. Minute beendet. Ab und zu treten noch einige tetanische Bewegungen und Krämpfe auf. Die Athmung ist sehr tief. Nach der 6. Minute erfolgt nochmals ein geringer tetanischer Anfall. Nach Verlauf desselben ist das Thier völlig ruhig.

13 Minuten nach der ersten Injection wird dem Thiere nochmals dieselbe Dosis Strychnin in derselben Weise in die Lungen eingespritzt. Nach der ersten Minute erfolgt bei Erschütterung des Brettes ein starker Tetanus. 11/2 Minuten: Auf Erschütterung des Brettes hin entwickelt sich ein deutlicher Opistotonus. Die Athmung steht. 13/4 Minuten: Die Athmung beginnt wieder. 2 Minuten nach der 2. Injection erfolgt spontaner Tetanus, kurz nachher lässt der Krampf wieder nach, die Athmung bleibt tief und frequent. 5 Minuten nach der Injection sind noch deutliche Strychninwirkungen beim Anklopfen auf das Brett oder Anblasen des Thieres sichtbar. Das Thier erholt sich jedoch bald und frisst bereits wieder nach 20 Minuten.

$$
[\ldots]
$$

\section{Versuch 16.}

Einem grossen Kaninchen (2450gr schwer) werden $5 \mathrm{cbcm}$ einer 0,25\%igen Curarelösung in die Lungen injicirt, sodass das Thier $0,0125 \mathrm{cbcm}$ Curare erhält. Sofort nach der Injection wird das Thier vom Brett losgeschnallt und aufrecht hingesetzt. 55 Sekunden nach der Injection werden die Pupillen enger. Nach 11/4 Minuten: Der Kopf sinkt nieder. 11/2 Minuten: Die Pupillen haben sich noch mehr verengt. Das Thier berührt mit dem Kinn den Tisch; die Athmung wird langsam. 2 Minuten: Der Kopf sinkt auf die Seite. 21/4 Minuten: Das Thier liegt ganz auf der Seite, die Athmung ist sehr langsam. 3 Minuten: Die Athembewegungen werden sehr schwach, 28 in der Minute, die Pupillen sind sehr eng; das Thier ist vollständig gelähmt; zeitweise treten Erschütterungen des ganzen Thieres auf. 5 Minuten: Die Athmung ist äusserst schwach, die Reflexthätigkeit aufgehoben. Die Pupillen erweitern sich fast plötzlich auf das Maximum. Die Athmung ist ganz minimal und nur an den Lungenpartieen noch wahrnehmbar. 7 Minuten nach der Injection tritt der Tod des Thieres ein.

$$
[\ldots]
$$

\section{Versuch 19. \\ $\nabla$}

Einem Kaninchen (1800 gr schwer) werden $5 \mathrm{ckm}$ einer 0,25\% igen Algen Atropinlösung von der Trachea aus direkt in die Lungen injicirt. Das Thier erhält also 0,0125 cbm Atropin. Die Wirkung des Atropins zeigt sich bereits nach 65 Sekunden. Die Pupillen werden grösser und reagiren nur noch ganz schwach.

Nach 11ㄹㄹ Minuten reagiren die Pupillen nicht mehr. Dieser Zustand hält noch eine Stunde an. Das Thier zeigt sonst keine abnormen Veränderungen.
Aus den obigen auf das sorgfältigste angestellten Versuchen ergiebt sich, dass die Resorption von seiten der Lungen eine auffallend rasche ist, sowohl bei den Versuchen, bei welchen der Nachweis der in die Lungen injicirten Substanz in dem Secret der Nieren geliefert wurde, als bei denjenigen, deren Ergebniss auf den allgemeinen Vergiftungserscheinungen basirt.

Was zunächst den Vergleich mit der Resorptionsgeschwindigkeit vom Magen aus anlangt, so ist die Aufsaugung von seiten der Lungen gegenüben den dort gewonnenen Resultaten eine so ungemein rasche, dass man dieselbe im Vergleich zu den vom Magen aus constatirten Verhältnissen als eine fast momentane Wirkung bezeichnen darf.

Wir finden in der Litteratur, dass z.B. Jodkalium vom gesunden Magen aus resorbirt, erst nach frühestens 10 Minuten im Harne nachweisbar ist.

Weiter sehen wir, dass z. B. Natrium salicylicum bei solcher Versuchsanordnung erst nach 20 bis 25 Minuten frühestens im Urin sich auffinden lässt; Ergebnisse, welche wir mit geringen Einschränkungen aus eigenen Erfahrungen bestätigen können.

Vergleichen wir hiermit die Resultate, welche wir durch Experimente gewonnen haben, so erhellt bei der ersten Gruppe unserer Versuche, dass durchschnittlich schon nach 1 Minute der Nachweis der durch die Lungen vollzogenen Resorption durch die Harnprobe gelingt. So lehrt z. B. der Versuch 10, dass zwischen der 1. und 2. Minute nach der Injection das Jodkalium deutlich im Harne nachgewiesen wird, ebenso Versuch 11. In den Versuchen 1 bis 3 liess sich bereits nach 1 Minute das Vorhandensein von Natrium salicylicum im Harne constatiren.

Wenn auch die Resorption vom Unterhautzellgewebe aus nicht derartige zeitliche Differenzen im Verhältniss zu der von den Lungen aus stattgehabten aufweist, so geht doch aus unseren Versuchen klar hervor, dass durch die Lungen gelöste Substanzen rascher in den Organismus übergeführt werden, als vom Unterhautzellgewebe aus (cf. Versuch 4 und 8).

Auch die zweite Gruppe unserer Untersuchungen liefert Resultate, welche mit den obigen im Einklange stehen. Der Kürze halber verweisen wir auf die Experimente selbst.

Wie weit nun aus diesen experimentellen Ergebnissen praktisch verwertbare Folgerungen sich ableiten, steht zur Zeit noch aus. Nur wenig Anhaltspunkte giebt die Literatur für diesen Gesichtspunkt, nämlich von den Lungen aus eine allgemeine therapeutische Wirkung für den gesammten Organismus zu erzielen.

Die Wege, auf denen wir eine derartige Wirkung erlangen können, sind ja bekanntlich sehr mannigfach und bequem; aber wenn es darauf ankommt, neben der allgemeinen Wirkung noch eine specielle zu erzielen, so wird man in concreten Fällen gewiss die auffallend grosse Resorptionsgeschwindigkeit der Lungen benutzen dürfen.

Von diesem Gesichtspunkt aus sind die Resultate Gerhard's ${ }^{11}$ anzusehen, welcher endocarditische Processe durch Inhalationen von Natrium bicarbonicum ( 0,5 bis 1,0\%ige Lösung) in sehr günstiger Weise beeinflusste. Es wird sich vielleicht noch der Mühe lohnen, in solch' geeigneten Fällen das von Gerhard zuerst angewandte Princip weiter zu verfolgen.

\footnotetext{
${ }^{11}$ Ziemssen, Handbuch der allgemeinen Therapie. 1. B. 4.

T. Oertel, Respiratorische Therapie. S. 50.
} 